\title{
The Origin of Jovian Planets in Protostellar Disks: The Role of Dead Zones
}

\author{
Soko Matsumura ${ }^{1} \&$ Ralph E. Pudritz ${ }^{2}$ \\ Dept. of Physics \& Astronomy, McMaster University, Hamilton, ON L8S 4M1, Canada
}

\begin{abstract}
The final masses of Jovian planets are attained when the tidal torques that they exert on their surrounding protostellar disks are sufficient to open gaps in the face of disk viscosity, thereby shutting off any further accretion. In sufficiently well-ionized disks, the predominant form of disk viscosity originates from the Magneto-Rotational Instability (MRI) that drives hydromagnetic disk turbulence. In the region of sufficiently low ionization rate - the so-called dead zone - turbulence is damped and we show that lower mass planets will be formed. We considered three ionization sources (X-rays, cosmic rays, and radioactive elements) and determined the size of a dead zone for the total ionization rate by using a radiative, hydrostatic equilibrium disk model developed by Chiang et al. (2001). We studied a range of surface mass density $\left(\Sigma_{0}=10^{3}-10^{5} \mathrm{~g} \mathrm{~cm}^{-2}\right)$ and X-ray energy $\left(k T_{x}=1-10 \mathrm{keV}\right)$. We also compared the ionization rate of such a disk by X-rays with cosmic rays and find that the latter dominate $\mathrm{X}$-rays in ionizing protostellar disks unless the X-ray energy is very high $(5-10 \mathrm{keV})$. Among our major conclusions are that for typical conditions, dead zones encompass a region extending out to several AU - the region in which terrestrial planets are found in our solar system. Our results suggest that the division between low and high mass planets in exosolar planetary systems is a consequence of the presence of a dead zone in their natal protoplanetary disks. We also find that the extent of a dead zone is mainly dependent on the disk's surface mass density. Our results provide further support for the idea that Jovian planets in exosolar systems must have migrated substantially inwards from their points of origin.
\end{abstract}

Subject headings: circumstellar matter - planetary system - solar system: formation accretion disks - MHD - stars: pre-main-sequence

\section{Introduction}

The discovery of exosolar Jovian-mass planets has stimulated intense efforts to understand how planets form (e.g. Lin et al. 1999; Wuchterl et al. 2000). The current theoretical models

\footnotetext{
${ }^{1}$ soko@physics.mcmaster.ca

${ }^{2}$ pudritz@physics.mcmaster.ca
} 
are based on two general types of physical processes - disk instability or core accretion. In the disk instability picture (e.g. Cameron 1978; Boss 1997; Mayer et al. 2002), gravitational instability in a sufficiently massive disk fragments it into the clumps out of which planets may form. This process may require as little as $\sim 10^{3}$ years to form Jovian planets, but may not be able to explain sub-Jupiter mass planets (Boss 2000). This is in stark contrast to the core accretion model (e.g. Mizuno 1980; Pollack et al. 1996) wherein the coagulation of dust grains ultimately leads to the formation of planetesimals that collide with one another to form planetary cores. When a core reaches a critical mass in the range $\sim 10-15 M_{\oplus}$, gas around the protoplanet quickly accretes onto it ("runaway accretion") forming a gas giant. This process takes at least $\sim 10^{6}-10^{7}$ years - the average disk lifetime (e.g., Feigelson \& Montmerle 1999), and may not be able to explain massive Jovian planets.

Our paper focuses on an important aspect of Jovian planet formation that is common to both pictures. Regardless of whether proto-Jovian planets form through core accretion or gravitational instability, accretion onto a planet will continue until it is massive enough for the tidal torque that it exerts upon the surrounding disk to overcome the viscous disk torque which continues to fill-in any gap (e.g. Lin \& Papaloizou 1985). Planets that are sufficiently massive to open gaps in the disks have final masses that depend upon the disk viscosity (Nelson et al. 2000). In absence of viscosity, the tidal torque induced by the protoplanet opens a gap sooner thereby terminating the accretion earlier and leaving the planet with a smaller mass.

Thus the final mass of Jovian planets comes down to the question of the origin of the viscosity of protostellar disks at the time that significant protoplanetary cores are present within them. If disks are turbulent, then turbulent viscosity is likely to be the major factor in determining these masses. The Magneto-Rotational Instability (MRI) is the most promising source of turbulent disk viscosity (Balbus \& Hawley 1991). Even in the presence of an initially very weak magnetic field, the MRI will generate a significantly magnetized disk. This MRI viscosity requires good coupling between the gas and the magnetic field. Poor coupling - which occurs when the gas is insufficiently ionized - damps out the MRI and leads to a so-called "dead zone" where the viscosity is effectively zero (Gammie 1996). The high column density for protoplanetary disks ensures that dead zones are expected to occur during planet formation (Sano et al. 2000).

Disk viscosity will not entirely vanish, even in the dead zone, however. The gravitational interaction between the fairly massive protoplanetary cores - the forerunners of the Jovian planets - and the disk generates density waves within the disk which can transport angular momentum and provide an effective "viscosity" in the disk. This disk-planet interaction leads to both planetary migration (Goldreich \& Tremaine 1980) as well as evolution of the disk through the nonlinear damping and shocking of the waves (Larson 1989; Goodman \& Rafikov 2001, - GR01). The deposition of the angular momentum back into the disk as a consequence of this shock-induced wave damping is equivalent to the action of a viscous process, (Spruit 1987; Larson 1989) with an equivalent dimensionless $\alpha$ parameter of the order $\alpha_{d a m p} \simeq 10^{-4}-10^{-3}$ (e.g. GR01). This viscosity parameter is one to two orders of magnitude smaller than is expected for MRI turbulence in the 
regions of the disk where the magnetic field is well coupled to the fluid.

We can readily compare the masses of planets that are predicted for each of these mechanisms of disk viscosity. For the case of a turbulent viscous disk (e.g. Bryden et al. 1999; Rafikov 2002), the gap will open when the angular momentum transfer rate by tidal torque $\dot{H}_{T}=0.23\left(M_{p} / M_{*}\right)^{2} \Sigma a_{p}^{4} \Omega^{2}\left(a_{p} / h\right)^{3}$ exceeds that driven by viscous torque $\dot{H}_{\nu}=3 \pi \Sigma \nu a_{p} \Omega$. Here $\Omega$ is the Keplerian angular velocity and $\nu$ is the viscosity. Expressing the viscosity in terms of a standard $\alpha$ parameter $\nu \sim \alpha_{\text {turb }} c_{s} h$ (Shakura \& Sunyaev 1973), the ratio of a gap opening planetary mass $M_{p}$ to a stellar mass $M_{*}$ is

$$
\frac{M_{p, t u r b}}{M_{*}} \lesssim \sqrt{40 \alpha_{\text {turb }}\left(\frac{h}{a_{p}}\right)^{5}} .
$$

where the disk height and the radius of the planet's orbit are $h$ and $a_{p}$, respectively.

In the case that disk viscosity is provided solely by damped density waves, then the ratio of the planetary masses has been calculated by Rafikov (2002);

$$
\frac{M_{p, d a m p}}{M_{*}} \lesssim \frac{2}{3}\left(\frac{h}{a_{p}}\right)^{3} \min \left[5.2 Q^{-5 / 7}, 3.8\left(Q \frac{a_{p}}{h}\right)^{-5 / 13}\right]
$$

where the Toomre parameter is $Q=\Omega c_{s} /(\pi G \Sigma), c_{s}$ is the sound speed, and $\Sigma$ is the surface mass density. Note that the gap opening condition for an inviscid disk by Lin \& Papaloizou (1985) corresponds to $Q=1$ case. For the standard disk model by Chiang et al. (2001), we calculate that $Q=54$ and $h / a_{p} \sim 0.04$ at $1 \mathrm{AU}$, so that the planetary mass is very small.

By taking the ratio of these two predicted planetary mass scales, we immediately see that the planetary masses in well coupled zones in which the MRI is active will be much larger than in the dead zone for which only the $\alpha_{d a m p}$ value pertains. As an example, at $1 \mathrm{AU}$, and for a Toomre $\mathrm{Q}$ value of 54 calculated above, the mass of a Jovian planet in a well-coupled zone dictated by MRI turbulence $M_{p, t u r b}$ will exceed the mass that a Jovian protoplanet will attain in a dead zone through density wave damping $M_{p, \text { damp }}$ provided that $\alpha_{\text {turb }}$ exceeds a minimum value of;

$$
\alpha_{\text {turb }}>\alpha_{\text {min }} \equiv \min \left[1.0 \times 10^{-3} \frac{h}{a_{p}}, 7.4 \times 10^{-3}\left(\frac{h}{a_{p}}\right)^{23 / 13}\right]
$$

which is less than $10^{-4}$ for the aspect ratio at $1 \mathrm{AU}\left(h / a_{p} \sim 0.04\right)$. Numerical simulations of the MRI instability show that $\alpha_{\text {turb }}$ is much larger than this - by two orders of magnitude or more. Thus, a distinct jump in planetary masses - by a factor that is similar to the ratio of the masses of the Jovian to terrestrial planets - is expected at the disk radius that determines the maximum radial extent of the dead zone.

In this paper, we perform a detailed investigation in order to calculate precisely where the dead zone occurs in well constrained protostellar disk models. This allows us to determine where in the disk that this "jump" in planetary masses is likely to occur. In our second paper (to be submitted), we calculate precisely what these planetary masses are. We proceed by carefully computing the 
ionization balance within the protostellar disk, since it is this that determines the coupling of the magnetic field to the disk and hence, where the MRI induced turbulence can be sustained. The main sources of ionization in a circumstellar disk are X-rays from the stellar plasma (probably produced by magnetospheric reconnection events) as well as cosmic rays from the outside of the stellar system. Glassgold et al. (2000) showed that X-ray ionization dominates cosmic ray ionization out to $\simeq 1000 \mathrm{AU}$ in an optically thin disk. We compared these ionization sources for the selfconsistent disk model developed by Chiang et al. (2001). We include radioactive elements as a global and constant ionization source. We used the disk model developed by Chiang et al. (2001) primarily because it reproduces the spectral energy distributions (SEDs) of two T-Tauri and three Herbig Ae stars extremely well.

Our major finding is that dead zones typically encompass the region where terrestrial planets are found in our solar system $(\lesssim 2.0-3.3 \mathrm{AU})$. The physical definition of a dead zone is reviewed in $\S 2$. We introduce the disk model of Chiang et al. (2001) and elucidate the connection between the disk model and the X-ray ionization rate (using Glassgold et al. 1997) in $\S 3$. We state our results in $\S 4$ and apply them to the well studied case of AA Tau in $\S 5$. The important symbols used in this paper are summarized in Table 1.

\section{Dead Zones in Disk Models}

A dead zone is the region in a disk where MRI turbulence is unsustainable due to poor coupling of the field to the disk. The absence of turbulence is what gives this region its negligible viscosity. Gammie (1996) showed that MRI turbulence will be damped at a physical scale of $\lambda$ whenever the local growth rate of the MRI instability $\left(\simeq V_{A} / \lambda\right)$ is balanced by the Ohmic diffusion at that scale $\left(\simeq \eta / \lambda^{2}\right)$. For complete damping at some radius of the disk, one requires that all the turbulence scales less than the local pressure scale height $h(a)$ are damped; i.e. $\lambda \lesssim h$. By equating these two time-scales, one finds that the MRI turbulence damps in regimes with a small magnetic Reynolds number:

$$
R_{e M}=\frac{V_{A} h}{\eta} \lesssim 1
$$

Here, $V_{A} \equiv B / \sqrt{4 \pi \rho} \simeq \alpha_{\text {turb }}^{1 / 2} c_{s}$ is the Alfvén speed, $\rho$ is the mass density, $c_{s}=\sqrt{k T / \mu m_{H}}$ is the sound speed ( $k$ is the Boltzmann constant, $T$ is the temperature, $\mu$ is the mean molecular weight, and $m_{H}$ is the mass of the hydrogen) and $\alpha_{\text {turb }}$ is the viscosity parameter that measures the strength of the turbulence (Shakura \& Sunyaev 1973). This formula is one of the most important in the paper because it is the condition that determines the dead zone region in a disk once the disk structure and ionization state are specified. The importance of the latter is readily established by noting that the Reynolds number in this equation is sensitive to the ionization of the disk, through its dependence on the diffusivity of the magnetic field, $\eta$, which takes the form;

$$
\eta=\frac{234}{x_{e}} T^{1 / 2} \mathrm{~cm}^{2} \mathrm{~s}^{-1}
$$


Table 1: Some Important Symbols

\begin{tabular}{|c|c|c|}
\hline Symbol & Meaning & Reference \\
\hline 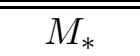 & Stellar mass & \\
\hline$R_{*}$ & Stellar radius & \\
\hline$R_{\odot}$ & Solar radius & \\
\hline$h$ & Pressure scale height of the disk & \\
\hline$H$ & Surface disk height & \\
\hline$a$ & Disk radius & \\
\hline$Q$ & Toomre's Q parameter: $Q=\Omega c_{s} /(\pi G \Sigma)$ & eq. (2) \\
\hline$c_{s}$ & Sound speed: $c_{s}=\sqrt{k T /\left(\mu m_{H}\right)}$ & $\S 1,2$ \\
\hline$\nu$ & Viscosity & $\S 1$ \\
\hline$\alpha_{\text {turb }}$ & $\alpha$ parameter of Shakura \& Sunyaev (1973) & $\S 1$ \\
\hline$V_{A}$ & Alfvén speed: $V_{A}=B / \sqrt{4 \pi \rho}$ & $\S 2$ \\
\hline$\eta$ & Magnetic diffusivity & eq. $(5)$ \\
\hline$R_{e M}$ & Magnetic Reynolds number & eq. $(4),(6),(7)$ \\
\hline$x_{e}$ & Electron fraction & eq. (8), (9) \\
\hline$x_{M}$ & Metal fraction & eq. (8) \\
\hline$\beta$ & Recombination coefficient & eq. (9) \\
\hline$\zeta$ & Ionization rate & eq. (8), (9), §3 \\
\hline$\zeta_{\text {tot }}$ & Net ionization rate: $\zeta_{t o t}=\zeta_{x}+\zeta_{C R}+\zeta_{R A}$ & eq. (10) \\
\hline$\zeta_{x}$ & $\mathrm{X}$-ray ionization rate & eq. (11) \\
\hline$\zeta_{C R}$ & Cosmic ray ionization rate & eq. (16) \\
\hline$\zeta_{R A}$ & Radioactive elements ionization rate & $\S 3$ \\
\hline$\sigma$ & Total photoelectric absorption cross section & eq. (14) \\
\hline$\tau$ & Optical depth & eq. (13) \\
\hline$d$ & Distance from the X-ray source to the disk & eq. (11) \\
\hline$n$ & Number density of neutral atoms & $\S 2$, eq. (17) \\
\hline$N_{H}$ & Surface number density along the path of X-rays & eq. $(13),(15)$ \\
\hline$N_{\perp}$ & Vertical surface number density: $N_{\perp}=\int_{z}^{\infty} n\left(a, z^{\prime}\right) d z^{\prime}$ & eq. (15) \\
\hline$\chi$ & Vertical surface mass density: $\chi=\int_{z}^{\infty} \rho\left(a, z^{\prime}\right) d z^{\prime}$ & eq. (16) \\
\hline$\alpha$ & Grazing angle from the central star & eq. $(21)$ \\
\hline$\alpha^{\prime}$ & Grazing angle from the X-ray source at $\left(12 R_{\odot}, 12 R_{\odot}\right)$ & eq. $(15),(21)$ \\
\hline
\end{tabular}


Table 2: Parameters used in the models.

\begin{tabular}{|c|c|c|c|c|c|l|}
\hline Fig. & $R_{e M, \text { crit }}$ & $\alpha_{\text {turb }}$ & $L_{x}\left[\mathrm{erg} \mathrm{s}^{-1}\right]$ & $k T_{x}[\mathrm{keV}]$ & $\Sigma_{0}\left[\mathrm{~g} \mathrm{~cm}^{-2}\right]$ & \multicolumn{1}{c|}{$\left[\mathrm{s}^{-1}\right]$} \\
\hline \hline 2 & 1 & $1,0.1,0.01$ & $10^{29}$ & 1 & $10^{3}$ & $\zeta=\zeta_{x}$ \\
3 & 1 & $1,0.1,0.01$ & No X-rays & No X-rays & $10^{3}$ & $\zeta=\zeta_{C R}$ \\
4 & 1 & $1,0.1,0.01$ & $10^{29}$ & 1 & $10^{3}$ & $\zeta=\zeta_{\text {tot }}$ \\
\hline 5 & 1 & 0.01 & $10^{29}$ & $1,2,3,5,10$ & $10^{3}, 5 \times 10^{3}, 10^{4}, 6 \times 10^{4}, 10^{5}$ & $\zeta=\zeta_{x}$ \\
6 & 1 & 0.01 & $10^{29}$ & $1,2,3,5,10$ & $10^{3}, 5 \times 10^{3}, 10^{4}, 6 \times 10^{4}, 10^{5}$ & $\zeta=\zeta_{\text {tot }}$ \\
7 & 1 & 0.01 & $10^{29}$ & $1,2,3,5,10$ & $10^{3}, 5 \times 10^{3}, 10^{4}, 6 \times 10^{4}, 10^{5}$ & $\zeta=\zeta_{x}$ \\
8 & 1 & 0.01 & $10^{29}$ & $1,2,3,5,10$ & $10^{3}, 5 \times 10^{3}, 10^{4}, 6 \times 10^{4}, 10^{5}$ & $\zeta=\zeta_{\text {tot }}$ \\
\hline 9 & 1 & $1,0.1,0.01$ & $0.439 \times 10^{30}$ & 1.21 & $1.5 \times 10^{3}$ & $\zeta=\zeta_{x}, \zeta_{C R}$ \\
10 & 1 & $1,0.1,0.01$ & $0.439 \times 10^{30}$ & 1.21 & $1.5 \times 10^{3}$ & $\zeta=\zeta_{\text {tot }}$ \\
\hline
\end{tabular}

Table 3: Dead zone radii for the star with the X-ray luminosity of $10^{29} \mathrm{erg} \mathrm{s}^{-1}$. The range of dead zone radius corresponds to the range of surface mass density.

\begin{tabular}{|c|c|c|c|c|c|}
\hline$\left(R_{e M, \text { crit }}, \alpha_{\text {turb }}\right)$ & cosmic rays $(\mathrm{CRs})$ & $k T_{x}=1 \mathrm{keV}$ & $k T_{x}=10 \mathrm{keV}$ & total $(1 \mathrm{keV}+\mathrm{CR})$ & total $(10 \mathrm{keV}+\mathrm{CR})$ \\
\hline \hline$(1,1)$ & $0.70-6.5 \mathrm{AU}$ & $2.7-29 \mathrm{AU}$ & $0.24-3.7 \mathrm{AU}$ & $0.70-6.5 \mathrm{AU}$ & $0.24-3.7 \mathrm{AU}$ \\
$(1,0.01)$ & $1.3-8.6 \mathrm{AU}$ & $3.9-41 \mathrm{AU}$ & $0.66-6.7 \mathrm{AU}$ & $1.3-8.6 \mathrm{AU}$ & $0.66-6.7 \mathrm{AU}$ \\
$(100,1)$ & $2.7-13 \mathrm{AU}$ & $6.3-62 \mathrm{AU}$ & $1.6-13 \mathrm{AU}$ & $2.7-13 \mathrm{AU}$ & $1.5-12 \mathrm{AU}$ \\
$(100,0.01)$ & $5.8-21 \mathrm{AU}$ & $10-97 \mathrm{AU}$ & $3.6-28 \mathrm{AU}$ & $5.8-21 \mathrm{AU}$ & $3.6-20 \mathrm{AU}$ \\
\hline
\end{tabular}


where $T$ is the disk temperature that is obtained from disk models (either interior $T_{i}$ or disk surface $T_{d s}$ depending on the disk height we are interested in - see $\S 2.3$, eq. (3) and (4) in Chiang et al. 2001). The electron fraction is $x_{e} \equiv n_{e} / n$ where $n_{e}$ and $n$ are the number density of electrons and neutral atoms respectively. The lower the electron fraction, the higher is the diffusivity coefficient for the field and hence the smaller is the magnetic Reynolds. Thus, poorly ionized disks will tend to have lower values of $R_{e M}$, resulting in larger dead zones.

One of the recent numerical MRI disk simulations by Fleming et al. (2000) defined the magnetic Reynolds number as

$$
R_{e M}^{\prime}=\frac{c_{s} h}{\eta},
$$

and determined the critical value $R_{e M, c r i t}^{\prime}=100$ assuming the presence of a uniform vertical magnetic field. Another recent analysis by Balbus \& Terquem (2001) suggested that the Hall effect should be included into the diffusion equation. The Hall effect is due to the velocity difference between electrons and ions, and causes a small transverse potential difference. The effect also diffuses the magnetic field just as the ohmic diffusion does. It implies that the magnetic Reynolds number might be smaller than the case of Fleming et al. (2000).

Summarizing these results, it appears that the critical value of magnetic Reynolds number $R_{e M, \text { crit }}$ is somewhat uncertain and that there are a few different definitions for $R_{e M}$. Taking these uncertainties into account, we used the critical magnetic Reynolds number of 1 and 100 with the alpha parameter of $1,0.1$, and 0.01 . The extreme cases of $\left(R_{e M, \text { crit }}, \alpha_{\text {turb }}\right)=(1,1),(1,0.01),(100$, 1 ), and $(100,0.01)$ correspond to $R_{e M}^{\prime}=1,10,100$, and 1000 respectively in the definition by Fleming et al. (2000). The following is the criterion we used to determine the dead zone:

$$
R_{e M}=\frac{V_{A} h}{\eta} \lesssim R_{e M, c r i t}
$$

The dead zone is the region in the disk where the magnetic Reynolds number $R_{e M}$ is smaller than some critical value $R_{e M \text {,crit }}=1$ or 100 . This region is characterized as having no MRI turbulence.

The calculation of the needed electron fraction $x_{e}$ (see eq. (5)) is well established, at least in principle. Assuming steady state ionization balance, Oppenheimer \& Dalgarno (1974) derived an equation for the electron fraction in the gas; namely,

$$
x_{e}^{3}+\frac{\beta_{t}}{\beta_{d}} x_{M} x_{e}^{2}-\frac{\zeta}{\beta_{d} n} x_{e}-\frac{\zeta \beta_{t}}{\beta_{d} \beta_{r} n} x_{M}=0,
$$

where $x_{M}$ is the metal fraction. In our case, $\zeta$ represents the ionization rate of X-rays, cosmic rays or radioactive elements, or total ionization rate of these three sources. Their mathematical forms are found in $\S 3$. Three beta terms represent three kinds of recombination rate coefficients - the dissociative recombination rate coefficient for electrons with molecular ions $\left(\beta_{d}=\right.$ $\left.2 \times 10^{-6} T^{-1 / 2} \mathrm{~cm}^{3} \mathrm{~s}^{-1}\right)$, the radiative recombination coefficient for electrons with metal ions $\left(\beta_{r}=3 \times 10^{-11} T^{-1 / 2} \mathrm{~cm}^{3} \mathrm{~s}^{-1}\right)$ and the rate coefficient of charge transfer from molecular ions to metal atoms $\left(\beta_{t}=3 \times 10^{-9} \mathrm{~cm}^{3} \mathrm{~s}^{-1}\right)$. 
In this paper, we only take account of the two extreme cases - the metal poor $\left(x_{M}=0\right)$ and the metal dominant $\left(x_{M} \gg x_{e}\right)$. In both cases, the electron fraction is written as

$$
x_{e}=\sqrt{\frac{\zeta}{\beta n}},
$$

where the number density $n$ depends on disk models (see eq. (17)) and $\beta$ is a recombination rate coefficient. In the metal poor case, only the recombination of electrons with molecular ions (dissociative recombination) is important and $\beta=\beta_{d}=2 \times 10^{-6} T^{-1 / 2} \mathrm{~cm}^{3} \mathrm{~s}^{-1}$. We use this coefficient throughout the paper except $\S 5$. In the metal dominant case, only the recombination of electrons with heavy metal ions (radiative recombination) becomes important and the recombination rate coefficient is replaced by the radiative recombination coefficient $\beta=\beta_{r}=3 \times 10^{-11} T^{-1 / 2} \mathrm{~cm}^{3} \mathrm{~s}^{-1}$ (Fromang et al. 2002). The astrophysical significance of these two cases is related to the density regime of the protostellar disk. We compare the results for these two cases as applied to AA Tau, in $\S 5$.

\section{Ionization Rate of a Self-Consistent Disk Model}

The previous section outlined precisely how the dead zone within a disk can be calculated once a disk model and ionization rate can be specified. In this section, we review the three sources of ionization for protostellar disks - X-rays from the central protostar, cosmic rays, and radioactive elements that are mixed with the disk material - and then calculate their respective ionization rates in the context of our chosen disk model. There is one caveat concerning cosmic ray ionization of protostellar disks - cosmic rays are likely to be swept away from an region containing a turbulent MHD jet or outflow (e.g. Skilling \& Strong 1976; Cesarsky \& Völk 1978). Since jets are manifest in Class I and II Young Stellar Objects (YSOs), it is possible that the bulk of the ionization of these sources is achieved solely by their YSO X-ray fluxes. For completeness, we show both sets of results - ionization from purely X-ray as well as combined ionization.

The combined contribution of each of these ionization sources produces the total ionization rate which is their sum;

$$
\zeta_{t o t}=\zeta_{x}+\zeta_{C R}+\zeta_{R A}
$$

We summarize the calculation of each of these three rates below and then also compare the effects of X-rays with that of cosmic rays in our disk model calculations.

\subsection{Ionization Rates}

The X-ray ionization rate was investigated by Krolik \& Kallman (1983) and further developed

by Glassgold et al. (1997). Glassgold et al. (2000) wrote the secondary electron contribution to the 
X-ray ionization rate $\zeta_{x}$ as follows:

$$
\zeta_{x}=\left[\left(\frac{L_{x}}{k T_{x} 4 \pi d^{2}}\right) \sigma\left(k T_{x}\right)\right]\left(\frac{k T_{x}}{\Delta \epsilon}\right) J\left(\tau, x_{0}\right),
$$

where $L_{x}$ is the observed X-ray luminosity, and $\sigma\left(k T_{x}\right)$ and $\tau\left(k T_{x}\right)$ are the total photoelectric absorption cross section and the optical depth at the energy $E=k T_{x}$ respectively. The distance between the X-ray source and some point of the disk surface is denoted by "d" and the energy to make an ion pair is $\Delta \epsilon$. In above equation, the first factor between the square brackets corresponds to the primary ionization rate, assuming the same energy $E=k T_{x}$ for all primary electrons. The second factor $k T_{x} / \Delta \epsilon$ then reads as the number of secondary electrons produced by a photoelectron with the energy $k T_{x}$. The last factor $J\left(\tau, x_{0}\right)$ represents the attenuation of X-rays. Using the dimensionless energy parameter $x=E / k T_{x}$, the attenuation factor $J\left(\tau, x_{0}\right)$ is written as

$$
J\left(\tau, x_{0}\right)=\int_{x_{0}}^{\infty} x^{-n} e^{-x-\tau\left(k T_{x}\right) x^{-n}} d x
$$

The optical depth $\tau\left(k T_{x}\right)$ measures how opaque the disk is toward the X-ray radiation from the star and is written as

$$
\tau\left(k T_{x}\right)=N_{H} \sigma\left(k T_{x}\right)
$$

where $\sigma\left(k T_{x}\right)$ is

$$
\sigma\left(k T_{x}\right)=\tilde{\sigma}_{0}\left(\frac{k T_{x}}{k e V}\right)^{-n} .
$$

We assumed that heavy elements are depleted onto grains and used $\tilde{\sigma}_{0}=8.50 \times 10^{-23} \mathrm{~cm}^{2}$ and $n=2.810$ (Glassgold et al. 1997). The surface number density $N_{H}$ is measured along the radiation path from the X-ray source. Letting $\alpha^{\prime}$ be the angle between the radiation path and the radial axis, the surface number density is

$$
\begin{aligned}
N_{H} & =\frac{N_{\perp}}{\sin \alpha^{\prime}} \\
& =\frac{\int_{z}^{\infty} n\left(a, z^{\prime}\right) d z^{\prime}}{\sin \alpha^{\prime}},
\end{aligned}
$$

where $N_{\perp}$ is the vertical surface density and $n(a, z)$ is the number density at the disk radius "a" and the height "z". Note that $n(a, z)$ and $\alpha^{\prime}$ depend on the disk model (see eq. (17) and (21) respectively).

Usually, the ionization rate by cosmic rays is estimated as $\zeta_{C R, 0} \simeq 10^{-17} \mathrm{~s}^{-1}$ (Spitzer \& Tomasko 1968), but it's not generally known to within better than an order of magnitude (Glassgold et al. 2000). In some cases, however, the cosmic-ray ionization rate is well constrained. As an example, van der Tak \& van Dishoeck (2000) obtained the cosmic-ray ionization rate of $2.6 \pm 1.8 \times 10^{-17} \mathrm{~s}^{-1}$ through $\mathrm{H}^{13} \mathrm{CO}$ submillimeter emission lines from massive protostars. This value is in good agreement with Voyager/Pioneer data $\left(4 \times 10^{-17} \mathrm{~s}^{-1}\right)$. Since the attenuation length for cosmic rays is 
$\chi_{C R} \sim 96 \mathrm{~g} \mathrm{~cm}^{-2}$ (Umebayashi \& Nakano 1981), we follow Sano et al. (2000) and write the cosmic ray ionization rate as follows

$$
\zeta_{C R}=\frac{\zeta_{C R, 0}}{2}\left(\exp \left[-\frac{\chi(a, z)}{\chi_{C R}}\right]+\exp \left[-\frac{\Sigma(a)-\chi(a, z)}{\chi_{C R}}\right]\right)
$$

where $\chi(a, z)$ is the vertical column density measured at some height $z\left(\chi(a, z)=\int_{z}^{\infty} \rho\left(a, z^{\prime}\right) d z^{\prime}\right)$.

The radioactive elements in the disk is yet another source of ionization, though they usually have only a minor effect on the total ionization rate. Following Umebayashi \& Nakano (1981), we use the ionization rate by radioactive elements of $\zeta_{R A}=6.9 \times 10^{-23} \mathrm{~s}^{-1}$.

\subsection{Disk Model}

Chiang \& Goldreich (1997), following Kenyon \& Hartmann (1987), developed a self-consistent passive disk model. Their model has a two-layer structure - a high temperature surface layer and a lower temperature interior. Dust grains in the surface layer of the disk absorb the flux of ultraviolet (UV) photons from the central star. Half of the emission from the grains in the surface layer escapes into the space and the remaining half heats the disk interior up. The model assumes the vertical hydrostatic equilibrium so that the number density may be expressed as

$$
n(a, z)=n_{0}(a) e^{-\frac{z^{2}}{2 h^{2}}},
$$

where the number density at the midplane $n_{0}$ is

$$
n_{0}(a)=\Sigma(a) /\left(\sqrt{2 \pi h(a)^{2}} \mu_{g}\right) .
$$

Here, $\mu_{g}=3 \times 10^{-24} \mathrm{~g}$ is the mean molecular weight of the gas, $\Sigma(a)$ is the surface density and $h(a)$ is the pressure scale height (Chiang et al. 2001):

$$
\begin{aligned}
\Sigma(a) & =\Sigma_{0}\left(\frac{a}{\mathrm{AU}}\right)^{-3 / 2} \\
h(a) & =\left(\frac{T_{i}}{T_{c}}\right)^{1 / 2}\left(\frac{a}{R_{*}}\right)^{1 / 2} a,
\end{aligned}
$$

where $\Sigma_{0}$ is the surface mass density at $1 \mathrm{AU}, T_{c} \equiv G M_{*} \mu_{g} / k R_{*}$ is a virial temperature that measures the gravitational potential at the surface of the central star, and $M_{*}$ and $R_{*}$ are the stellar mass and radius respectively. Note that the disk interior temperature $T_{i}$ (the disk temperature below the pressure scale height "h") decreases with a disk radius, so $h(a)$ increases slightly weaker than the power of three halves (see eq. (20)) and $n_{0}(a)$ decreases slightly weaker than the power

of three (see eq. (18)). The pressure scale height $h(a)$ is the height measured from the midplane to the interior disk, not the surface layer of the disk.

The ionization of this disk by cosmic rays and radioactive elements is straight-forward to calculate. The X-ray ionization requires that we model the geometry of the source of the X-rays 
- the central young stellar object. X-ray emission is thought to arise from the reconnection of large-scale magnetized loops, that are possibly but not necessarily related to the magnetopause radius of the stellar magnetic field and the inner edge of the disk. Accordingly, we place the X-ray source in this picture at a fiducial distance of $12 R_{\odot}$ above the disk and $12 R_{\odot}$ away from the central star following Glassgold et al. (1997).

The X-rays from this source graze the surface of the flaring disk and penetrate it to produce the X-ray induced ionization. It is convenient to relate this grazing angle, $\equiv \alpha^{\prime}$, to the grazing angle defined in the disk model of Chiang et al. (2001) $\equiv \alpha$ (see eq. (5) in Chiang et al. 2001). Assuming that the average radiation from the central star originates at a distance $R_{*} / 2$ from the disk plane on the stellar surface (Hartmann 2000), we write the grazing angle from the X-ray source (see eq. (15)) as

$$
\begin{aligned}
\alpha^{\prime}= & \alpha-\beta+\gamma \\
\beta & \equiv \tan ^{-1}\left(\frac{d \ln H}{d \ln a} \frac{H}{a}\right) \\
\gamma & \equiv \tan ^{-1}\left(\frac{H-\frac{1}{2} R_{*}}{a-\frac{\sqrt{3}}{2} R_{*}}\right)-\tan ^{-1}\left(\frac{H-12 R_{\odot}}{a-12 R_{\odot}}\right),
\end{aligned}
$$

where $H$ is the height of the surface layer of the disk and defined as $H / h \equiv 4$ in Chiang et al. (2001). Fig. 1 is a schematic figure of this equation. The grazing angle from the X-ray source, $\alpha^{\prime}$ is the angle between the disk midplane and the solid arrow. The first term of the righthand side of the equation, $\alpha$ is the angle between the disk surface and the dashed arrow of the UV emission from the star. The second term $\beta$ shows the flaring angle of the disk and the third term $\gamma$ comes from the geometry of the X-ray source. Note that $\gamma=0$ corresponds to an X-ray source that is at the stellar surface.

We calculated the X-ray ionization rate (eq. (11)) by integrating the attenuation factor (eq. (12)) using MATLAB. Instead of an infinite upper limit of the energy, we used $x=100$ for the upper limit of the integration. For the lower limit, we used $x=1 \mathrm{keV} / k T_{x}(E=1 \mathrm{keV})$, following Glassgold et al. (1997). The surface number density along the path of the radiation $N_{H}$ found in the optical depth (eq. (13)) is calculated by using the grazing angle $\alpha^{\prime}$ (eq. (21)) and the number density $n(a, z)$ that is obtained from the disk model of Chiang et al. (2001) (see eq. (17) and (18)). Note that this number density $n(a, z)$ depends on the disk interior temperature $T_{i}$ and the pressure scale height of the disk $h$. Both of them are determined by solving the radiative equilibrium equations (3) and (4) in Chiang et al. (2001).

\section{Results}

In this section, we study the extent of dead zones for various disks. We show that X-rays and cosmic rays are comparable ionization sources and determine the size of dead zones from the total ionization rate. Throughout this section, we assume the metal poor disk ( $\operatorname{set} \beta=\beta_{d}$ in eq. (9)). 


\subsection{Standard Disk Model}

First, we show the results of a fiducial model which uses the standard disk model of Chiang et al. (2001), the critical magnetic Reynolds number $R_{e M, \text { crit }}=1$ with $\alpha_{\text {turb }}=1,0.1$, 0.01, and when X-rays exist, the X-ray luminosity of $L_{x}=10^{29} \mathrm{erg} \mathrm{s}^{-1}$ with the temperature of $k T_{x}=1$ $\mathrm{keV}$. We chose the standard X-ray luminosity of $L_{x}=10^{29} \mathrm{erg} \mathrm{s}^{-1}$ because typical young stars have X-ray luminosities $L_{x} \sim 10^{28}-10^{30} \mathrm{erg} \mathrm{s}^{-1}$. Though the standard disk of Chiang et al. (2001) has the surface mass density at $1 \mathrm{AU}$ of $\Sigma_{0}=10^{3} \mathrm{~g} \mathrm{~cm}^{-2}$, we don't call this minimum mass solar nebular model because these two models are qualitatively different from each other. With the standard disk model of Chiang et al. (2001), we calculated the surface number density (eq. (15)) and/or the surface mass density ( $\chi$ in eq. (16)) to estimate the X-ray and/or cosmic ray ionization rate at each radius and height of the $2 \mathrm{D}$ disk. All disk parameters are the same as in Table 1 of Chiang et al. (2001). Other parameters used in this subsection is summarized in Table 2 in this paper. We determined the critical height of the dead zone at each radius of the disk from eq. (7).

The size of dead zones arising solely from X-ray irradiation are plotted in Fig. 2. The figure shows the vertical cross section of the standard disk. The region below the dashed, long-dashed and dot-dashed lines show the dead zones. As the magnetic field becomes weaker (the alpha value changes $\left.\alpha_{\text {turb }}=1,0.1,0.01\right)$, the dead zone becomes larger. The result follows from the fact that as the magnetic field becomes weak, the growth rate of the MRI instability is reduced with respect to the local damping rate (see eq. (4) and the text therein).

The equivalent result for cosmic rays is shown in Fig. 3. In this case, we just replaced the ionization rate in electron fraction (eq. (9)) with the cosmic ray ionization rate calculated by eq. (16). Again, we find the smaller dead zone as the magnetic field becomes stronger.

These two figures show that cosmic rays dominate $1 \mathrm{keV}$ X-rays in ionizing a disk. The dead zone stretches out to $\sim 5-7 \mathrm{AU}$ in the case of illumination by X-rays and only to $\sim 1-2 \mathrm{AU}$ in cosmic ray case.

The dividing line between X-ray or cosmic ray ionized regions may be found by equating their ionization rates. We call this resulting vertical scale height the Röntgen height. Note that due to the higher number density, the X-ray ionization rate decreases toward the disk midplane as does the electron fraction, while the cosmic ray ionization rate is about the same everywhere in the disk. X-ray ionization dominates cosmic ray ionization above the Röntgen height.

Fig. 4 shows the dead zones estimated by the total ionization rate. Again, we took a surface mass density at $1 \mathrm{AU}$ of $\Sigma_{0}=10^{3} \mathrm{~g} \mathrm{~cm}^{-2}$, the dead zone criterion $R_{e M} \leq 1$, an X-ray luminosity of $L_{x}=10^{29} \mathrm{erg} \mathrm{s}^{-1}$, and an X-ray energy of $k T_{x}=1 \mathrm{keV}$. The Röntgen height suggests that X-rays dominate cosmic rays only in the surface layer - where the disk is optically thin. Comparing Fig. 3 with 4 , it is apparent that the size of dead zones are determined by cosmic rays for the standard disk case.

There are two characteristic dead zone radii. One of them is the midplane radius where the 
dead zone surface cuts the disk midplane. The other one is the fiducial radius where the dead zone surface crosses the pressure scale height of the interior disk. We take this latter case to be our "fiducial" value because planets are likely to form in the interior disk that has much higher density (the order of $\left.\sim 10^{3}\right)$ and larger particles $(0.01-1000 \mu \mathrm{m}$ instead of $0.01-1 \mu \mathrm{m})$ compared to the surface layers.

\subsection{Sensitivity of Dead Zone Radius to Model Parameters}

Next, we compare cases of different parameters $\left(\Sigma_{0}, k T_{x}\right)$ with one another. This is done to reveal which parameter has the largest effects on the size of dead zone. We also present results for two cases; one for only X-ray induced ionization, and the other for ionization arising from the total ionization rate. We make this distinction in order that the contribution of X-rays can be clearly discerned (cf. discussion at the beginning of $\S 5$ ). Throughout this subsection, we set $R_{e M, c r i t}=1$ and $\alpha_{\text {turb }}=0.01$ (see Table 2). These are the conditions suggested by, for example, Gammie (1996).

Fig. 5 is the plot of the fiducial dead zone radius estimated by X-ray alone as a function of the X-ray energy $\left(k T_{x}=1,2,3,5\right.$, and $\left.10 \mathrm{keV}\right)$. For the X-ray ionization calculation, we used the typical X-ray luminosity of $L_{x}=10^{29} \mathrm{erg} \mathrm{s}^{-1}$. We include the minimum mass solar nebula $\left(\Sigma_{0}=10^{3} \mathrm{~g} \mathrm{~cm}^{-2}\right)$ as well as a very massive disk of $\Sigma_{0}=10^{5} \mathrm{~g} \mathrm{~cm}^{-2}$ suggested by Murray et al. (1998) for Jupiter to migrate from 5 AU to $<1$ AU. It is apparent that the sizes of dead zones decrease as the X-ray energy increases and/or the disk surface mass density decreases.

Note that the figure depends on the choice of the critical magnetic Reynolds number $R_{e M, c r i t}$, the alpha parameter $\alpha_{\text {turb }}$, and the X-ray luminosity $L_{x}$. For example, if we increase the Xray luminosity by two orders of magnitude $\left(L_{x}=10^{31} \mathrm{erg} \mathrm{s}^{-1}\right)$ in Fig. 5, then the resulting curves are pushed down by the factor of 10 . This is because of the definition of the magnetic Reynolds number $R_{e M}=\sqrt{\alpha_{\text {turb }} \zeta}\left[c_{s} h /(234 \sqrt{\beta n T})\right]$. Here, the bracket term is determined by a disk model (i.e. independent of the X-ray energy or luminosity) so that the magnetic Reynolds number is changed depending on $\alpha_{\text {turb }}$ and $\zeta$. For X-rays, the ionization rate is written as $\zeta_{x}=L_{x}\left[\sigma\left(k T_{x}\right) /\left(4 \pi d^{2} \Delta \epsilon\right) J\left(\tau, x_{0}\right)\right]$ where the luminosity changes the result by two orders of magnitude in our case (we used $L_{x}=10^{29} \mathrm{erg} \mathrm{s}^{-1}$ and $10^{31} \mathrm{erg} \mathrm{s}^{-1}$ ).

The results for the dead zone radius for a disk undergoing the total ionization rate is shown in Fig. 6. It shows that the radii of dead zones are almost constant for X-ray energy of $k T_{x}=1-3 \mathrm{keV}$ and coincide with the results of Fig. 5 in the higher X-ray energy region.

These figures also show the obvious point that the size of dead zones is not affected by low energy X-rays $\left(k T_{x} \sim 1-3 \mathrm{keV}\right)$ and that the dead zone gets smaller as the surface mass density decreases. The decrease of the surface mass density (from upper lines to lower ones) leads to a smaller optical depth $\tau$ and a larger ionization rate $\zeta$, so that the dead zone becomes smaller.

We summarize the effect of changing parameters. If the X-ray luminosity is larger $\left(10^{31} \mathrm{erg} \mathrm{s}^{-1}\right.$ 
rather than $10^{29} \mathrm{erg} \mathrm{s}^{-1}$ ), X-rays penetrate deeper in the disk and therefore the dead zone becomes smaller. If the critical magnetic Reynolds number is larger (100 rather than 1), a very small magnetic field diffusivity can destroy the turbulence - the viscosity becomes negligible and therefore the dead zone tends to be larger. If the alpha parameter is smaller ( 0.01 rather than 1$)$, then the magnetic field is weaker, turbulent viscosity is smaller and the dead zone becomes larger. In short, to have a smaller dead zone, a disk has to have a smaller surface mass density $\Sigma_{0}$, a larger X-ray luminosity $L_{x}$ and a larger $\alpha_{\text {turb }}$ value as well as a smaller critical Reynolds number $R_{e M, c r i t}$.

To draw the reader's attention to our major result, we present Fig. 7 and 8 and study the effect of the surface mass density at $1 \mathrm{AU} \Sigma_{0}$. Fig. 7 uses the same data sets as Fig. 5, but here we plot the fiducial dead zone radius as a function of the surface mass density at $1 \mathrm{AU}$ $\left(\Sigma_{0}=10^{3}, 5 \times 10^{3}, 10^{4}, 6 \times 10^{4}\right.$, and $\left.10^{5} \mathrm{~g} \mathrm{~cm}^{-2}\right)$ instead of the X-ray energy. It is apparent that if cosmic rays penetrate disks without being swept away, they dominate X-rays in ionizing a disk except for $k T_{x} \sim 5-10 \mathrm{keV}$.

Fig. 8 shows the equivalent result for the total ionization rate and uses the same data sets as Fig. 6. The X-rays have a rather small effect on the size of dead zones even when they have high energy $\left(k T_{x} \sim 5-10 \mathrm{keV}\right)$. The most striking result is that dead zone radii vary by at most an order of magnitude, from $1-10 \mathrm{AU}$ for a range of two orders of magnitude in disk surface density and an order of magnitude in the X-ray energy. The robustness of this result indicates that the region of Jovian-mass planet formation is rather similar for a wide variety of protostellar disk systems.

We can see that the differences between lines in Fig. 7, 8 are smaller than those in Fig. 5, 6. It appears that the size of the dead zone is most sensitive to the surface mass density and, to a lesser extent, the X-ray energy. These figures also show that the dead zone produced by cosmic rays is usually smaller than that by X-rays. X-rays dominate cosmic rays in ionizing the circumstellar disk only when the X-ray source has a high energy $\left(k T_{x} \sim 5-10 \mathrm{keV}\right)$.

Table 3 shows the size range of dead zones for four extreme cases $-\left(R_{e M, \text { crit }}, \alpha_{\text {turb }}\right)=(1,1)$, $(1,0.01),(100,1)$, and $(100,0.01)$. The minimum and maximum dead zone radii correspond to the minimum and maximum surface mass density at $1 \mathrm{AU}$. It is apparent that the dead zone is sensitive not only to the surface mass density or X-ray energy, but also to the value of the magnetic Reynolds number. The important point of this table is that the dead zone radii for total ionization rate are about the same as the region of terrestrial planets in our solar system except the one extreme case. For this extreme case of $\left(R_{e M, c r i t}, \alpha_{t u r b}\right)=(100,0.01)$, which is equivalent to $R_{e M, c r i t}^{\prime}=1000$ or $\left(R_{e M, \text { crit }}, \alpha_{\text {turb }}\right)=\left(1,10^{-6}\right)$, the dead zone gets pushed out to $5.8 \mathrm{AU}$.

Finally, we note that the data presented in Figures 5, 7 and 8 corresponding to the variation of dead zone radii can be well fit by approximate linear curves. For completeness, we present these fits in Appendix A - Table 5, 6, 7, and 8. 


\section{Application to the case of AA Tau}

We now specialize our model to an observed protostellar disk - AA Tau. We consider this source because of the excellent fit of the Chiang et al. model to the data from this system. The stellar and disk parameters that we used are listed in Table 4 and are taken from Chiang et al. (2001) other than X-ray parameters. The X-ray luminosity $L_{x}$ and the X-ray energy $k T_{x}$ are taken from Neuhäuser et al. (1995). For the dead zone criterion, we used $R_{e M, \text { crit }}=1$ with $\alpha_{\text {turb }}=1,0.1,0.01$. These parameters are summarized in Table 2.

Fig. 9 is the estimated dead zone for AA Tau. It shows that X-ray ionization dominates cosmic ray ionization only in the surface layer where the optical depth is very small (see Röntgen height). The dead zone becomes smaller as the magnetic field gets stronger because of the stronger viscous torque that it exerts. In all cases the dead zones by cosmic rays are smaller than those produced by X-rays.

If the disk of AA Tau is metal dominant, the dead zone gets smaller. Fig. 10 shows the dead zones estimated by total ionization rate for both metal poor and metal dominant cases. Since metal ions recombine with electrons slowly, the electron fraction for metal dominant case is larger (compare $\beta_{d}$ with $\beta_{r}$ ) $-R_{e M}$ tends to be larger - and hence the dead zone is smaller. Note that the recombination coefficient of metals is $\sim 5$ orders of magnitude smaller than that of molecular ions. The "real" dead zone would be calculated by solving eq. (8) directly and would be located somewhere between these two extremes.

\section{Discussion and Conclusions}

We calculated the dead zones for a variety of disk models, as well as the specific case of a Class II source, AA Tau. This kind of calculation has been done by several authors (e.g., Gammie 1996; Igea \& Glassgold 1999; Sano et al. 2000; Fromang et al. 2002), but all of them use either the minimum mass solar nebula model developed by Hayashi et al. (1985) (e.g., Gammie 1996; Igea \& Glassgold 1999; Sano et al. 2000) or the $\alpha$ disk model developed by Shakura \& Sunyaev (1973) (Fromang et al. 2002). All our disk models are obtained by using the self-consistent passive disk model of Chiang et al. (2001). To calculate X-ray ionization rates, we followed the method used by Glassgold et al. (2000), while for cosmic rays, we used the method adopted by Sano et al. (2000). When the ionization rate becomes sufficiently low so that the magnetic Reynolds number is less than some critical value (1 or 100), we determined the size of the dead zone. We compared the

result by X-rays with cosmic rays and also obtained the size of the dead zone determined by the total ionization rate.

The fact that our dead zones extend as far as the region of terrestrial planet formation in our solar system is very important. In the accretion picture, considerable gas accretion must occur onto a sufficiently massive protoplanetary core. This must inevitably take place in the turbulent 
region of the disk beyond the dead zone. The major implication of our work is that the division between Jovian and sub-Jovian or terrestrial planets may be due to the presence of a dead zone. We explore the significance of these results for predicting planetary masses in a forthcoming paper. Our basic results are as follows:

1. Our major finding is that the typical dead zone encompasses, in physical scale, the terrestrial planets in our solar system.

A typical dead zone size estimated from the total ionization rate is $\sim 0.24-2.7$ AU for the fiducial disk. For an extreme case of $\left(R_{e M, \text { crit }}, \alpha_{\text {turb }}\right)=(100,0.01)\left(R_{e M, \text { crit }}^{\prime}=1000\right.$ or $\left(R_{e M, \text { crit }}, \alpha_{\text {turb }}\right)=\left(1,10^{-6}\right)$ equivalently $)$, the dead zone extends out to $\sim 5.8 \mathrm{AU}$. These results suggest that the observed exosolar Jovian-mass planets must have migrated to their observed positions from points of origin farther out in disk radius, beyond these dead zone radii.

2. X-rays dominate cosmic rays in ionizing a disk only in the surface of the disk for most cases.

Glassgold et al. (2000) noted that X-ray ionization dominates cosmic ray ionization out to $\simeq 1000 \mathrm{AU}$. We found that the disks are usually too optically thick for this to be generally true. $\mathrm{X}$-rays only dominate cosmic rays in the surface layer of the disk. Cosmic rays determine the size of the dead zone under typical conditions (compare, for example, Fig. 3 with Fig. 4).

3. Sufficiently high energy X-rays could dominate cosmic rays in ionizing a disk.

The X-rays could dominate the cosmic rays if the $\mathrm{X}$-ray energy is high $k T_{x}=5-10 \mathrm{keV}$ (compare Fig. 7 with 8). This is much higher energy for most observed sources however.

4. The size of a dead zone is sensitive to the disk surface mass density $\Sigma_{0}$ and the X-ray energy $k T_{x}$.

We found that the size of the dead zone is most sensitive to the surface mass density, and to a lesser extent, the X-ray energy (see Fig. $5 \& 6$ and Fig. $7 \&$ 8). The X-ray luminosity, the critical magnetic Reynolds number, the alpha viscous parameter however, have a significant effect on the size of a dead zone.

5. There is a power law relation between the size of a dead zone estimated by X-rays alone and $\Sigma_{0}$ or $k T_{x}$ (see Appendix).

The dead zone radius has power-law relations with both the surface mass density and the X-ray energy. The dead zone radii estimated by X-rays obey $a_{d} \propto \Sigma_{0}^{0.44}-\Sigma_{0}^{0.59}$ and $a_{d} \propto k T_{x}^{-0.46}-k T_{x}^{-1.1}$, while those estimated by cosmic rays obey $a_{d} \propto \Sigma_{0}^{0.28}-\Sigma_{0}^{0.49}$. The dead zone radii determined by the total ionization rate has the relation of $a_{d} \propto \Sigma_{0}^{0.28}-\Sigma_{0}^{0.59}$.

We thank Norm Murray, Joe Weingartuer and Steve Balbus for stimulating conversations on these topics. We also thank an anonymous referee for useful review of the manuscript. SM is supported by McMaster University, while REP is supported by a grant from the National Science 
and Engineering Research Council of Canada (NSERC).

\section{REFERENCES}

Balbus, S. A. \& Hawley, J. F. 1991, ApJ, 376, 214

Balbus, S. A. \& Terquem, C. 2001, ApJ, 552, 235

Boss, A. P. 1997, Science, 276, 1836

—. 2000, Disks, Planetesimals, and Planets (ASP Conference Series, 2000)

Bryden, G., Chen, X., Lin, D. N. C., Nelson, R. P., \& Papaloizou, J. C. B. 1999, ApJ, 514, 344

Cameron, A. G. W. 1978, Moon and Planets, 18, 5

Cesarsky, C. J. \& Völk, H. J. 1978, A\&A, 70, 367

Chiang, E. I. \& Goldreich, P. 1997, ApJ, 490, 368

Chiang, E. I., Joung, M. K., Creech-Eakman, M. J., Qi, C., Kessler, J. E., Blake, G. A., \& van Dishoeck, E. F. 2001, ApJ, 547, 1077

Feigelson, E. D. \& Montmerle, T. 1999, ARA\&A, 37, 363

Fleming, T. P., Stone, J. M., \& Hawley, J. F. 2000, ApJ, 530, 464

Fromang, S. ., Terquem, C., \& Balbus, S. A. 2002, MNRAS, 329, 18

Gammie, C. F. 1996, ApJ, 457, 355

Glassgold, A. E., Feigelson, E. D., \& Montmerle, T. 2000, Protostars and Planets IV (The University of Arizona Press, 2000)

Glassgold, A. E., Najita, J., \& Igea, J. 1997, ApJ, 480, 344

Goldreich, P. \& Tremaine, S. 1980, ApJ, 241, 425

Goodman, J. \& Rafikov, R. R. 2001, ApJ, 552, 793

Hartmann, L. 2000, Accretion Processes in Star Formation (Cambridge University Press, 2000)

Hayashi, C., Nakazawa, K., \& Nakagawa, Y. 1985, in Protostars and Planets II, 1100-1153

Igea, J. \& Glassgold, A. E. 1999, ApJ, 518, 848

Kenyon, S. J. \& Hartmann, L. 1987, ApJ, 323, 714

Krolik, J. H. \& Kallman, T. R. 1983, ApJ, 267, 610 
Larson, R. B. 1989, in The Formation and Evolution of Planetary Systems, 31-48

Lin, D. N. C., Bryden, G., \& Ida, S. 1999, in Astrophysical Discs - An EC Summer School, Astronomical Society of the Pacific, Conference series Vol \#160, Edited by J. A. Sellwood and Jeremy Goodman, 1999, p. 207., 207-+

Lin, D. N. C. \& Papaloizou, J. 1985, in Protostars and Planets II, 981-1072

Mayer, L., Quinn, T., Wadsley, J., \& Stadel, J. 2002, Science, 298, 1756

Mizuno, H. 1980, Progress of Theoretical Physics, 64, 544

Murray, N., Hansen, B., Holman, M., \& Tremaine, S. 1998, Science, 279, 69

Nelson, R. P., Papaloizou, J. C. B., Masset, F., \& Kley, W. 2000, MNRAS, 318, 18

Neuhäuser, R., Sterzik, M. F., Schmitt, J. H. M. M., Wichmann, R., \& Krautter, J. 1995, A\&A, 297,391

Oppenheimer, M. \& Dalgarno, A. 1974, ApJ, 192, 29

Pollack, J. B., Hubickyj, O., Bodenheimer, P., Lissauer, J. J., Podolak, M., \& Greenzweig, Y. 1996, Icarus, 124,62

Rafikov, R. R. 2002, ApJ, 572, 566

Sano, T., Miyama, S. M., Umebayashi, T., \& Nakano, T. 2000, ApJ, 543, 486

Shakura, N. I. \& Sunyaev, R. A. 1973, A\&A, 24, 337

Skilling, J. \& Strong, A. W. 1976, A\&A, 53, 253

Spitzer, L. J. \& Tomasko, M. G. 1968, ApJ, 152, 971

Spruit, H. C. 1987, A\&A, 184, 173

Umebayashi, T. \& Nakano, T. 1981, PASJ, 33, 617

van der Tak, F. F. S. \& van Dishoeck, E. F. 2000, A\&A, 358, L79

Wuchterl, G., Guillot, T., \& Lissauer, J. J. 2000, Protostars and Planets IV, 1081 
Table 4: Input parameters for AA Tau

\begin{tabular}{|c|c|c|}
\hline Symbol & Meaning & Standard value \\
\hline \hline$M_{*}$ & stellar mass & $0.67 M_{\odot}$ \\
$R_{*}$ & stellar radius & $2.1 R_{\odot}$ \\
$T_{*}$ & stellar effective temperature & $4000 \mathrm{~K}$ \\
$L_{x}$ & X-ray luminosity & $0.439 \times 10^{30} \mathrm{erg} \mathrm{s}^{-1}$ \\
$k T_{x}$ & X-ray energy & $1.21 \mathrm{keV}$ \\
\hline$T_{s u b}^{\text {iron }}$ & iron sublimation temperature & $2000 \mathrm{~K}$ \\
$T_{s u b}^{\text {sil }}$ & silicate sublimation temperature & $1500 \mathrm{~K}$ \\
$T_{s u b}^{\text {ice }}$ & ice $\mathrm{H}_{2} \mathrm{O}$ sublimation temperature & $150 \mathrm{~K}$ \\
\hline$\Sigma_{0}$ & surface mass density at 1 AU & $1.5 \times 10^{3} \mathrm{~g} \mathrm{~cm}{ }^{-2}$ \\
$p$ & $-d \log \Sigma / d \log a$ & 1.5 \\
$a_{i}$ & inner disk radius & $2 R_{*}$ \\
$a_{o}$ & outer disk radius & 3.8 \\
$H / h$ & visible photosphere height $/$ gas scale height & 3.5 \\
$q_{i}$ & $-d \log N / d \log r$ in the interior disk & 3.5 \\
$q_{s}$ & $-d \log N / d \log r$ in the surface disks & $1000 \mu \mathrm{m}$ \\
$r_{\text {max }, i}$ & maximum grain radius in the interior disk & $1 \mu \mathrm{m}$ \\
$r_{\text {max }, s}$ & maximum grain radius in the surface disks & $R_{*}=250 \mathrm{AU}$ \\
\hline
\end{tabular}




\section{Appendix A}

\section{$\mathrm{X}$-rays and Cosmic rays}

We supply tables of X-ray and cosmic ray power-law relations separately for interested readers. These approximate relations are all obtained by using the least square fitting.

Table 5 shows the fitting parameters for the dead zone radius by X-rays as a function of X-ray energy $k T_{x}$. For the same choice of the critical magnetic Reynolds number $R_{e M \text {,crit }}$ and the alpha parameter $\alpha_{\text {turb }}$, it is apparent that the curves show roughly the same power. One of the examples is shown in Fig. 5.

Table 6 shows the fitting parameters for the dead zone radius by X-rays as a function of the surface mass density at $1 \mathrm{AU}, \Sigma_{0}$. Again, we can see the similar power for the same choice of the critical magnetic Reynolds number $R_{e M \text {,crit }}$ and the alpha parameter $\alpha_{\text {turb }}$. One of the examples is shown in Fig. 7.

Table 7 shows the fitting parameters for the dead zone radius by cosmic rays as a function of the surface mass density at $1 \mathrm{AU}, \Sigma_{0}$. One of the examples is shown in Fig. 7 (the gray solid line with crosses).

Table 8 shows the fitting parameters for the dead zone radius estimated by the total ionization rate as a function of the surface mass density at $1 \mathrm{AU}, \Sigma_{0}$. Again, we can see the similar power for the same choice of the critical magnetic Reynolds number $R_{e M, \text { crit }}$ and the alpha parameter $\alpha_{\text {turb }}$. One of the examples is shown in Fig. 8. In particular, $k T_{x}=1-3 \mathrm{keV}$ give a very similar power law relation. 
Table 5: The sizes of dead zones as a function of $k T_{x}: y=a x^{b}$. The star has X-ray luminosity of $10^{29} \mathrm{erg} \mathrm{s}^{-1}$.

\begin{tabular}{|c|c|c|c|c|}
\hline$\left(R_{e M, \text { crit }}, \alpha_{\text {turb }}\right)$ & $\Sigma_{0}\left[\mathrm{~g} \mathrm{~cm}^{-2}\right]$ & $a$ & $b$ & $\chi^{2}$ \\
\hline \hline$(1,1)$ & $10^{3}$ & 2.67 & -1.05 & $0.692 e-4$ \\
& $5 \times 10^{3}$ & 5.97 & -0.908 & $0.583 e-3$ \\
& $10^{4}$ & 8.47 & -0.887 & $0.140 e-2$ \\
& $6 \times 10^{4}$ & 27.3 & -0.887 & $0.140 e-2$ \\
& $10^{5}$ & 28.6 & -0.883 & $0.164 e-2$ \\
\hline$(1,0.01)$ & $10^{3}$ & 3.73 & -0.776 & $0.179 e-2$ \\
& $5 \times 10^{3}$ & 8.58 & -0.761 & $0.201 e-2$ \\
& $10^{4}$ & 11.9 & -0.751 & $0.213 e-2$ \\
& $6 \times 10^{4}$ & 29.7 & -0.772 & $0.267 e-2$ \\
& $10^{5}$ & 38.5 & -0.786 & $0.238 e-2$ \\
\hline$(100,1)$ & $10^{3}$ & 5.86 & -0.609 & $0.403 e-2$ \\
& $5 \times 10^{3}$ & 12.8 & -0.619 & $0.321 e-2$ \\
& $10^{4}$ & 18.4 & -0.640 & $0.320 e-2$ \\
& $6 \times 10^{4}$ & 44.8 & -0.665 & $0.370 e-2$ \\
& $10^{5}$ & 57.9 & -0.676 & $0.296 e-2$ \\
\hline$(100,0.01)$ & $10^{3}$ & 9.59 & -0.458 & $0.450 e-2$ \\
& $5 \times 10^{3}$ & 20.8 & -0.483 & $0.503 e-2$ \\
& $10^{4}$ & 29.4 & -0.508 & $0.383 e-2$ \\
& $6 \times 10^{4}$ & 69.7 & -0.529 & $0.387 e-2$ \\
& $10^{5}$ & 89.5 & -0.544 & $0.423 e-2$ \\
\hline
\end{tabular}


Table 6: The sizes of dead zones as a function of $\Sigma_{0}: y=a x^{b}$. The star has the X-ray luminosity of $10^{29} \mathrm{erg} \mathrm{s}^{-1}$.

\begin{tabular}{|c|c|c|c|c|}
\hline$\left(R_{e M, \text { crit }}, \alpha_{\text {turb }}\right)$ & $k T_{x}[\mathrm{keV}]$ & $a$ & $b$ & $\chi^{2}$ \\
\hline \hline$(1,1)$ & 1 & $0.761 e-1$ & 0.515 & $0.315 e-4$ \\
& 2 & $0.359 e-1$ & 0.522 & $0.443 e-3$ \\
& 3 & $0.225 e-1$ & 0.530 & $0.658 e-3$ \\
& 5 & $0.123 e-1$ & 0.545 & $0.220 e-2$ \\
& 10 & $0.454 e-2$ & 0.589 & $0.820 e-2$ \\
\hline$(1,0.01)$ & 1 & 0.118 & 0.508 & $0.483 e-4$ \\
& 2 & $0.676 e-1$ & 0.501 & $0.977 e-4$ \\
& 3 & $0.488 e-1$ & 0.500 & $0.258 e-3$ \\
& 5 & $0.330 e-1$ & 0.502 & $0.477 e-3$ \\
& 10 & $0.213 e-1$ & 0.501 & $0.757 e-3$ \\
\hline$(100,1)$ & 1 & 0.201 & 0.498 & $0.496 e-4$ \\
& 2 & 0.131 & 0.483 & $0.178 e-5$ \\
& 3 & $0.995 e-1$ & 0.483 & $0.178 e-5$ \\
& 5 & $0.775 e-1$ & 0.476 & $0.474 e-4$ \\
& 10 & $0.652 e-1$ & 0.460 & $0.324 e-4$ \\
\hline$(100,0.01)$ & 1 & 0.369 & 0.483 & $0.178 e-5$ \\
& 2 & 0.256 & 0.471 & $0.158 e-4$ \\
& 3 & 0.212 & 0.467 & $0.387 e-4$ \\
& 5 & 0.187 & 0.455 & $0.469 e-4$ \\
& 10 & 0.171 & 0.441 & $0.723 e-4$ \\
\hline
\end{tabular}

Table 7: The sizes of dead zones produced by cosmic rays, as a function of $\Sigma_{0}: y=a x^{b}$.

\begin{tabular}{|c|c|c|c|}
\hline$\left(R_{e M, \text { crit }}, \alpha_{\text {turb }}\right)$ & $a$ & $b$ & $\chi^{2}$ \\
\hline \hline$(1,1)$ & $0.234 e-1$ & 0.485 & $0.978 e-3$ \\
$(1,0.01)$ & $0.650 e-1$ & 0.420 & $0.160 e-2$ \\
$(100,1)$ & 0.236 & 0.341 & $0.209 e-2$ \\
$(100,0.01)$ & 0.830 & 0.278 & $0.411 e-3$ \\
\hline
\end{tabular}


Table 8: The sizes of dead zones estimated from the total ionization rate as a function of $\Sigma_{0}$ : $y=a x^{b}$. The star has the X-ray luminosity of $10^{29} \mathrm{erg} \mathrm{s}^{-1}$.

\begin{tabular}{|c|c|c|c|c|}
\hline$\left(R_{e M, \text { crit }}, \alpha_{\text {turb }}\right)$ & $k T_{x}[\mathrm{keV}]$ & $a$ & $b$ & $\chi^{2}$ \\
\hline \hline$(1,1)$ & 1 & $0.234 e-1$ & 0.485 & $0.978 e-3$ \\
& 2 & $0.234 e-1$ & 0.485 & $0.978 e-3$ \\
& 3 & $0.217 e-1$ & 0.492 & $0.842 e-3$ \\
& 5 & $0.125 e-1$ & 0.539 & $0.854 e-3$ \\
& 10 & $0.454 e-2$ & 0.589 & $0.820 e-2$ \\
\hline$(1,0.01)$ & 1 & $0.650 e-1$ & 0.420 & $0.160 e-2$ \\
& 2 & $0.650 e-1$ & 0.420 & $0.160 e-2$ \\
& 3 & $0.617 e-1$ & 0.425 & $0.110 e-2$ \\
& 5 & $0.403 e-1$ & 0.464 & $0.415 e-4$ \\
& 10 & $0.213 e-1$ & 0.501 & $0.757 e-3$ \\
\hline$(100,1)$ & 1 & 0.236 & 0.341 & $0.209 e-2$ \\
& 2 & 0.224 & 0.346 & $0.153 e-2$ \\
& 3 & 0.202 & 0.356 & $0.699 e-3$ \\
& 5 & 0.136 & 0.391 & $0.158 e-3$ \\
& 10 & $0.753 e-1$ & 0.439 & $0.352 e-3$ \\
\hline$(100,0.01)$ & 1 & 0.830 & 0.278 & $0.411 e-3$ \\
& 2 & 0.730 & 0.290 & $0.125 e-3$ \\
& 3 & 0.591 & 0.310 & $0.393 e-3$ \\
& 5 & 0.443 & 0.336 & $0.483 e-3$ \\
& 10 & 0.278 & 0.374 & $0.439 e-3$ \\
\hline
\end{tabular}




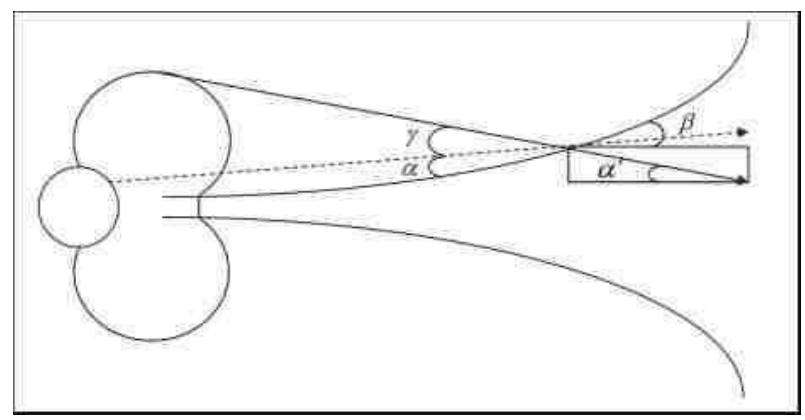

Fig. 1. - A schematic figure of the cross section of the disk. The solid arrow shows the X-rays from the magnetic field (the X-ray source is at $\left(12 R_{\odot}, 12 R_{\odot}\right)$ ) and the dashed arrow shows the light from the central star. Note that there is a relation of $\alpha^{\prime}=\alpha-\beta+\gamma$ among angles (see eq. (21) and the text).

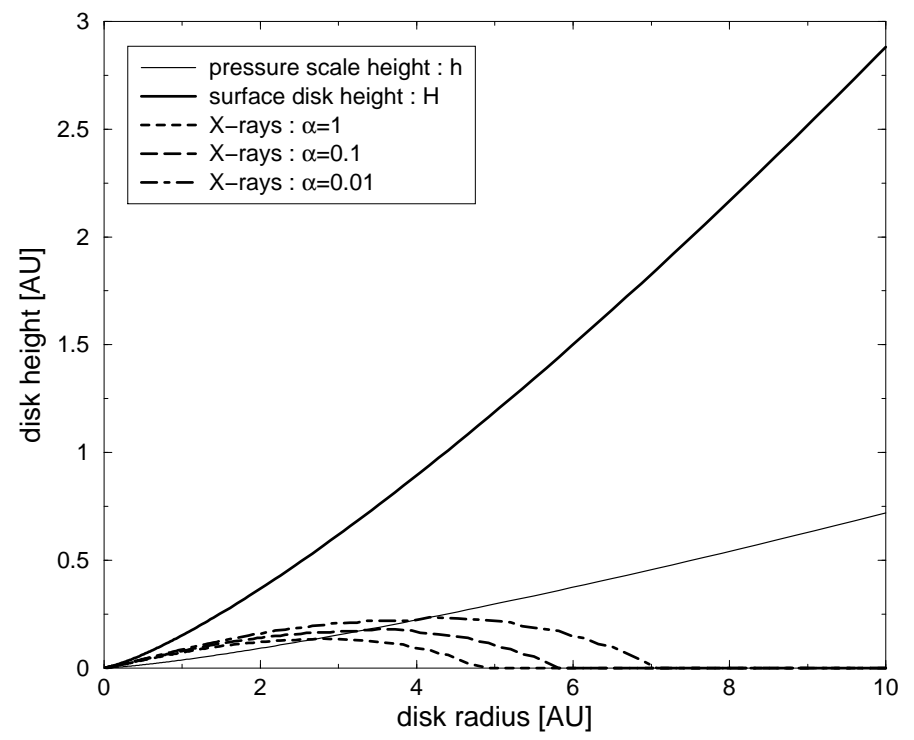

Fig. 2. - The dead zone estimated by X-rays for the standard disk model of Chiang et al. (2001). We used $L_{x}=10^{29} \mathrm{erg} \mathrm{s}^{-1}, k T_{x}=1 \mathrm{keV}, R_{e M, \text { crit }}=1$ and $\alpha_{\text {turb }}=1,0.1, \& 0.01$. X-ray dead zone stretches out to $5-7 \mathrm{AU}$. 


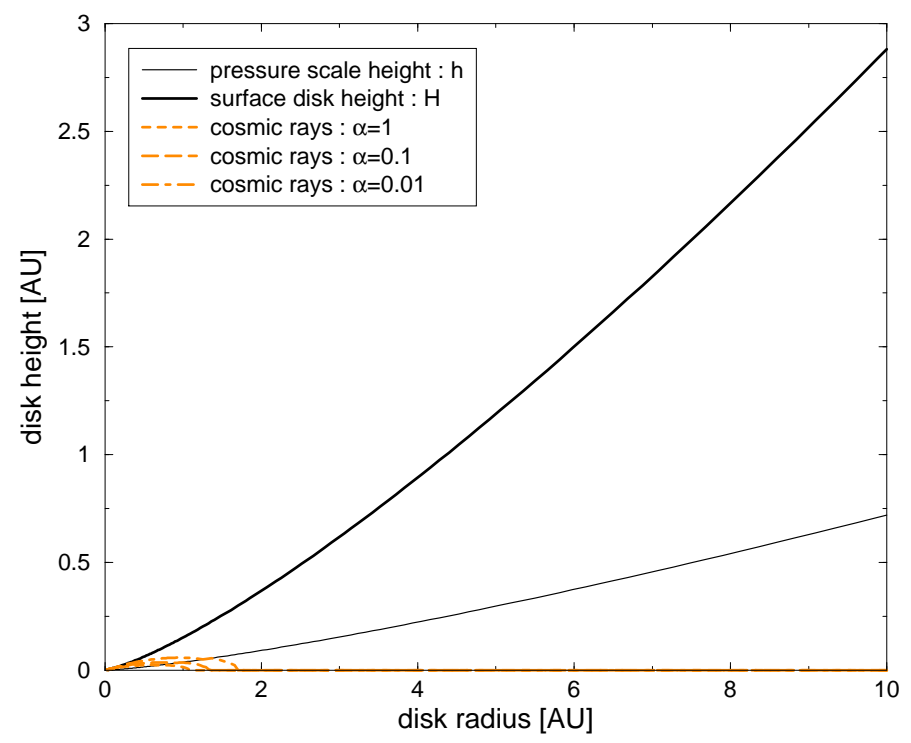

Fig. 3.- The dead zone estimated by cosmic rays for the standard disk model of Chiang et al. (2001). We used $R_{e M, c r i t}=1$ and $\alpha_{\text {turb }}=1,0.1$, \& 0.01. Cosmic ray dead zone stretches out to $1-2 \mathrm{AU}$.

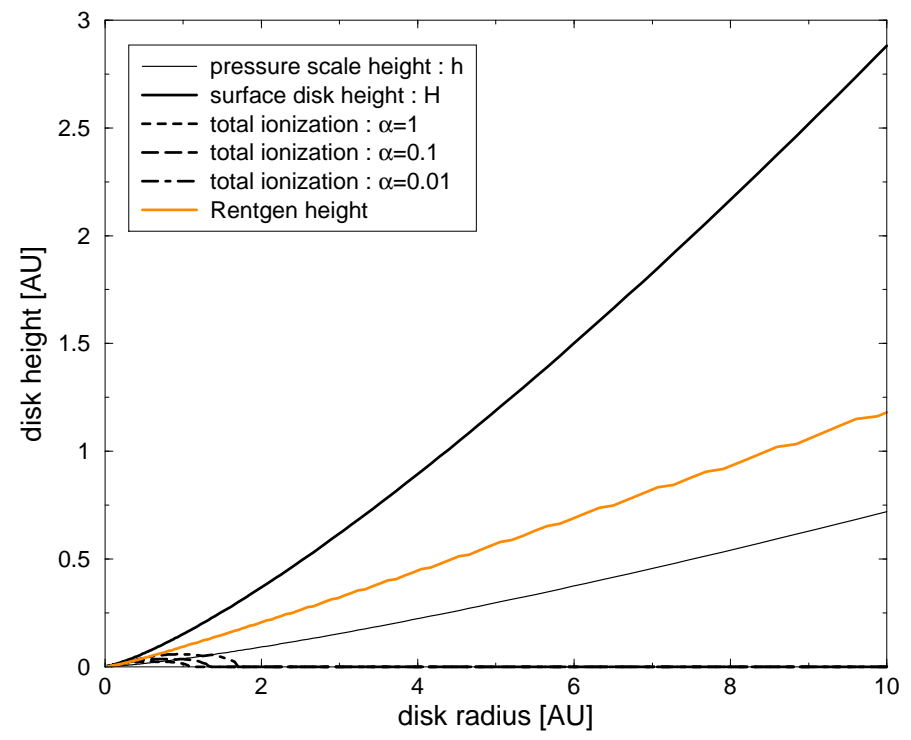

Fig. 4.- The dead zone determined by the total ionization rate for the standard disk model of Chiang et al. (2001). We used $L_{x}=10^{29} \mathrm{erg} \mathrm{s}^{-1}, k T_{x}=1 \mathrm{keV}, R_{e M, \text { crit }}=1$ and $\alpha_{\text {turb }}=$ $1,0.1, \& 0.01$. Also shown is the Röntgen height. X-rays dominate cosmic rays in ionizing a disk only at the surface layer, so the dead zone estimated from the total ionization rate has the same size as that from cosmic rays. 


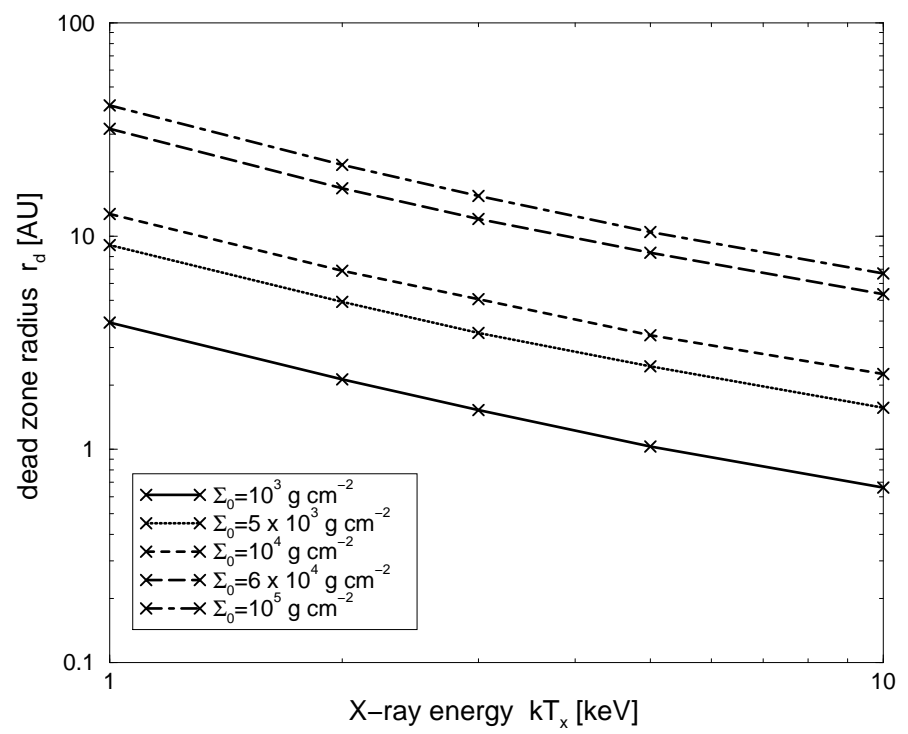

Fig. 5.- The fiducial dead zone radius as a function of the X-ray energy for a source luminosity $L_{x}=10^{29} \mathrm{erg} \mathrm{s}^{-1}$. We chose $R_{e M, c r i t}=1$ and $\alpha_{\text {turb }}=0.01$ and estimated the dead zone sizes by X-rays alone. Higher X-ray energy and smaller surface mass density lead to the smaller dead zone.

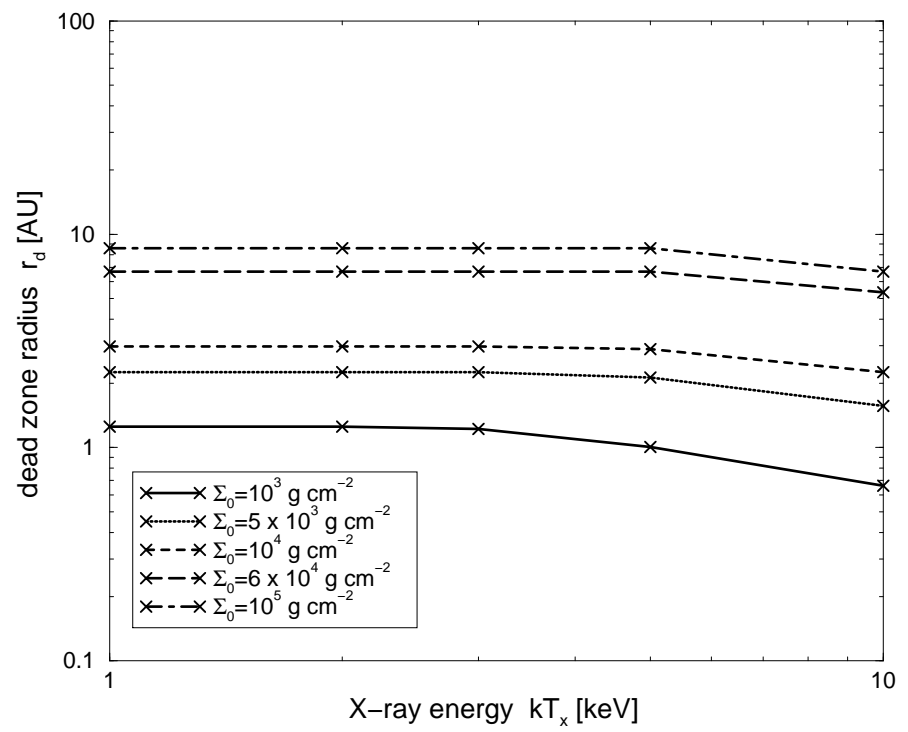

Fig. 6. - The fiducial dead zone radius as a function of the X-ray energy for a source luminosity $L_{x}=10^{29} \mathrm{erg} \mathrm{s}^{-1}$. We chose $R_{e M, c r i t}=1$ and $\alpha_{\text {turb }}=0.01$ and estimated the dead zone sizes by total ionization rates. Cosmic rays dominate low energy X-rays in ionizing a disk. 


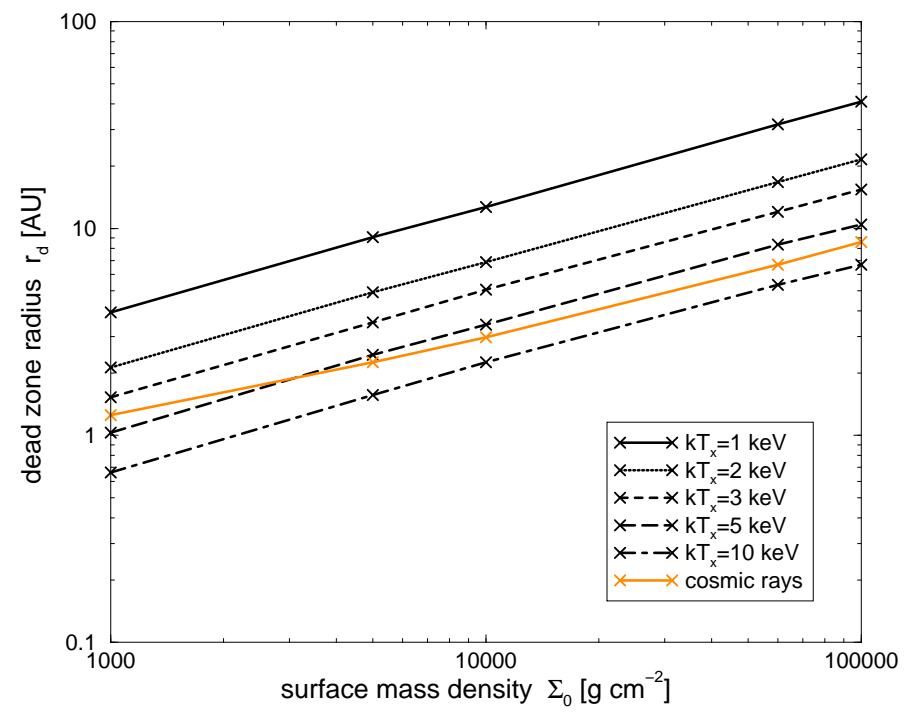

Fig. 7.- The fiducial dead zone radius as a function of the surface mass density for an X-ray luminosity $L_{x}=10^{29} \mathrm{erg} \mathrm{s}^{-1}$. We chose $R_{e M, \text { crit }}=1$ and $\alpha_{\text {turb }}=0.01$ and estimated the dead zone sizes by X-rays alone. Also shown is the dead zone radius estimated by cosmic rays. High energy $\mathrm{X}$-rays dominate cosmic rays in ionizing a disk.

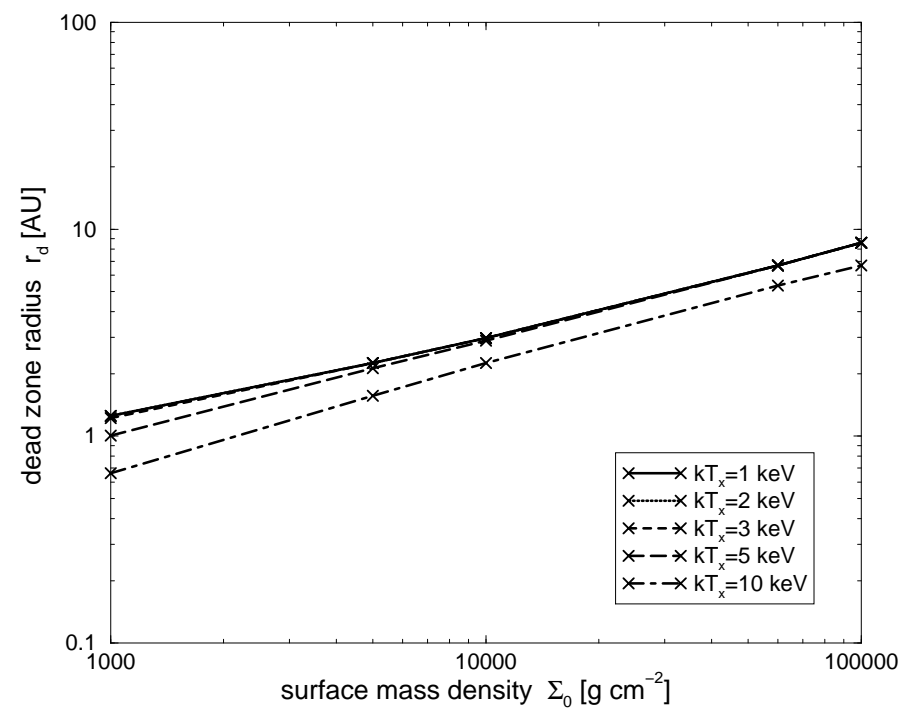

Fig. 8.- The fiducial dead zone radius as a function of the surface mass density for an X-ray luminosity $L_{x}=10^{29} \mathrm{erg} \mathrm{s}^{-1}$. We chose $R_{e M, \text { crit }}=1$ and $\alpha_{\text {turb }}=0.01$ and estimated the dead zone sizes by total ionization rates. The dead zone radius changes about an order of magnitude for a range of two orders of magnitude in disk surface mass density. 


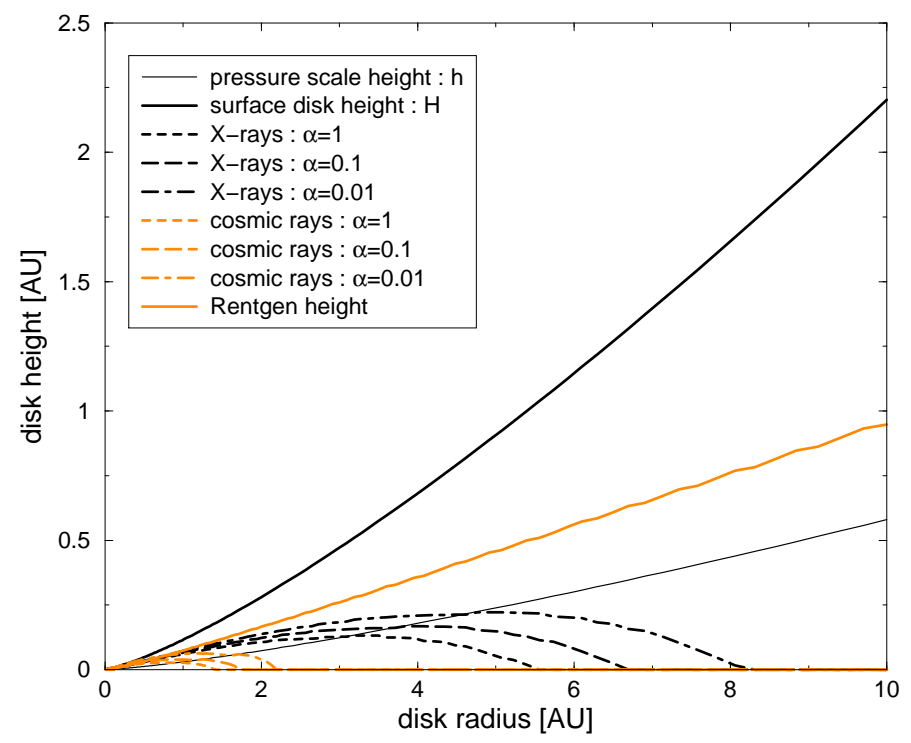

Fig. 9. - The dead zone of AA Tau estimated from X-rays is compared with that predicted from cosmic rays. We used $L_{x}=0.439 \times 10^{30} \mathrm{erg} \mathrm{s}^{-1}, k T_{x}=1.21 \mathrm{keV}, R_{e M, \text { crit }}=1$, and $\alpha_{\text {turb }}=$ $1,0.1$, and 0.01 . Also shown is the Röntgen height. Cosmic rays dominate X-rays in ionization almost throughout the disk.

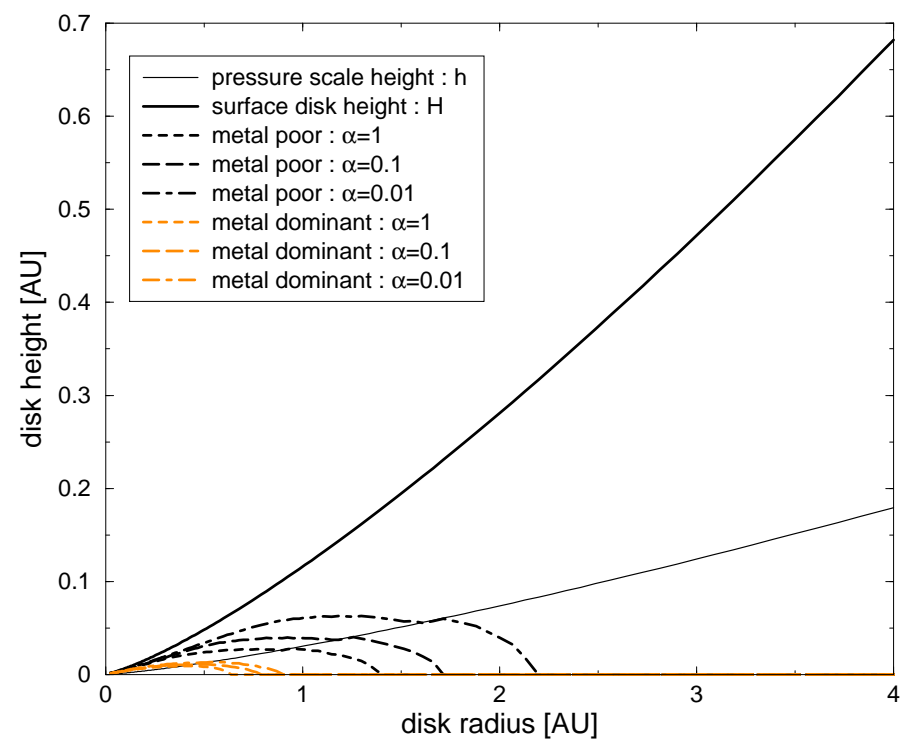

Fig. 10. - The dead zone of AA Tau estimated both for a metal poor and a metal dominant disk. We used $L_{x}=0.439 \times 10^{30} \mathrm{erg} \mathrm{s}^{-1}, k T_{x}=1.21 \mathrm{keV}, R_{e M, \text { crit }}=1$, and $\alpha_{\text {turb }}=1,0.1$, and 0.01 . The presence of metals makes the dead zone smaller. 